\title{
A Genetic Algorithm for Hybrid VANETs With Synchronous Communication
}

\author{
João M. Sarubbi \\ CEFETMG \\ Belo Horizonte, MG, Brazil \\ joao@decom.cefetmg.br \\ Cristiano M. Silva \\ UFSJ \\ Ouro Branco-MG, Brazil \\ cristiano@ufsj.edu
}

\author{
Flavio C. Martins \\ CEFETMG \\ Belo Horizonte, MG, Brazil \\ flaviocruzeiro@decom.cefetmg.br \\ Elizabeth Wanner \\ Aston University \\ Birmingham, UK \\ efwanner@gmail.com
}

\begin{abstract}
In this work, we propose a genetic algorithm for solving the allocation of Roadside Units (RSUs) in a Hybrid Vehicular Network with Synchronous Communication. We run our algorithm for several $\mathrm{V} 2 \mathrm{~V}$ communication ranges and compare the influence of these ranges in the number of chosen RSUs.
\end{abstract}

\section{CCS CONCEPTS}

-Computing methodologies $\rightarrow$ Heuristic function construction; •Mathematics of computing $\rightarrow$ Evolutionary algorithms;

\section{KEYWORDS}

VANETs, Hybrid Communication, Genetic Algorithm

\section{ACM Reference format:}

João M. Sarubbi, Flavio C. Martins, Cristiano M. Silva, and Elizabeth Wanner. 2017. A Genetic Algorithm for Hybrid VANETs With Synchronous Communication. In Proceedings of GECCO '17 Companion, Berlin, Germany, fuly 15-19, 2017, 2 pages.

DOI: http://dx.doi.org/10.1145/3067695.3076031

\section{INTRODUCTION}

Vehicular Networks [2] (VANETs) are a particular type of mobile network, which is specially designed to the domain of vehicles and pedestrians. In the last years, these networks have received considerable attention from the research community, as well as the automotive industry.

In a Vehicular Network, the communication may happen in three major ways: (i) Vehicle-to-Vehicle (V2V) [1], a pure wireless ad hoc network that in which communication is performed from vehicle to vehicle; (ii) Vehicle-to-Infrastructure (V2I) or Infrastructure-toVehicle (I2V) [5], an architecture with wired backbone and wireless last hops, in which the communication occurs through connections between vehicles and communication units, called Roadside Units (RSUs); and, (iii) Hybrid, communication that exploits the V2I and the V2V communications.

Permission to make digital or hard copies of part or all of this work for personal or classroom use is granted without fee provided that copies are not made or distributed for profit or commercial advantage and that copies bear this notice and the full citation on the first page. Copyrights for third-party components of this work must be honored. For all other uses, contact the owner/author(s).

GECCO '17 Companion, Berlin, Germany

(C) 2017 Copyright held by the owner/author(s). 978-1-4503-4939-0/17/07 ..\$15.00 DOI: http://dx.doi.org/10.1145/3067695.3076031
In this work, we propose a new genetic algorithm, called DeltaGA2, for solving the allocation of RSUs to guarantee a Delta Network [5]. The Delta Network is a metric designed to reflect the connectivity experienced by vehicles. Delta is based on two measurements: (i) connectivity duration $\left(\rho_{1}\right)$; and, (ii) percentage of vehicles presenting such connectivity duration $\left(\rho_{2}\right)$.

Different from Delta-GA algorithm proposed by Sarubbi et al. [4], this new algorithm allows the communication between vehicles within a V2V communication range $(\lambda)$. Our algorithm also allows the multi-hop communication. We test our algorithm for different $\lambda$ values to measure the impact to use a hybrid network instead of using a simple V2I network.

\section{PROBLEM DEFINITION}

A Deployment is $\Delta_{\rho_{2}}^{\rho_{1}}$ whenever $\rho_{2}$ percent of all vehicles must be connected to roadside units during $\rho_{1}$ percent of the trip. Formally:

Definition 2.1 (Deployment $\Delta_{\rho_{2}}^{\rho_{1}}$ ). Let $R$ represent a road network, and $V=\left\{v_{1}, v_{2}, \ldots, v_{n}\right\}$ represent the set of vehicles traveling on $R$. Let $C \subset V$ be the set of vehicles experiencing connection during, at least, $\rho_{1}$ percent of the trip duration. A deployment is considered $\Delta_{\rho_{2}}^{\rho_{1}}$ whenever $\frac{|C|}{|V|} \geq \rho_{2}$.

\section{GENETIC ALGORITHM}

In this section, we present our genetic algorithm[3], called DeltaGA2, to solve the Deployment $\Delta_{\rho_{2}}^{\rho_{1}}$ with hybrid network and synchronous communication. Our algorithm is based on the Delta-GA algorithm proposed by Sarubbi et al. [4]. Our algorithm has a standard structure with the Evaluation, Crossover and Mutation steps. We also implement the Elitism.

\subsection{Encoding}

In this work, we represent an individual as a list of coordinates $(\mathrm{x}, \mathrm{y})$ to install RSUs. The size of this list defines the number of RSUs present in the solution. Figure 1 represents an example of one individual with 8 RSUs.

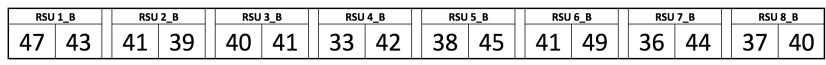

Figure 1: Representation of an individual with 8 RSUs 


\subsection{Decoding}

The individual decodification process allows computing both V2V and I2V communication. The Algorithms Decoding Procedure and Verify V2V Communication Procedure show our decoding strategy. Being possible the communication between a vehicle and an RSU in a particular time, the Add I $\mathrm{V}$ Communication Time procedure is responsible for adding this communication time in the vehicle travel. The Verify V2V Communication procedure verifies, for a given vehicle in a specific time unit all V2V communication possibility using a recursive strategy. Similarity from RSU communication, the Add V2V Communication Time procedure is responsible for adding the V2V communication time in the vehicle travel.

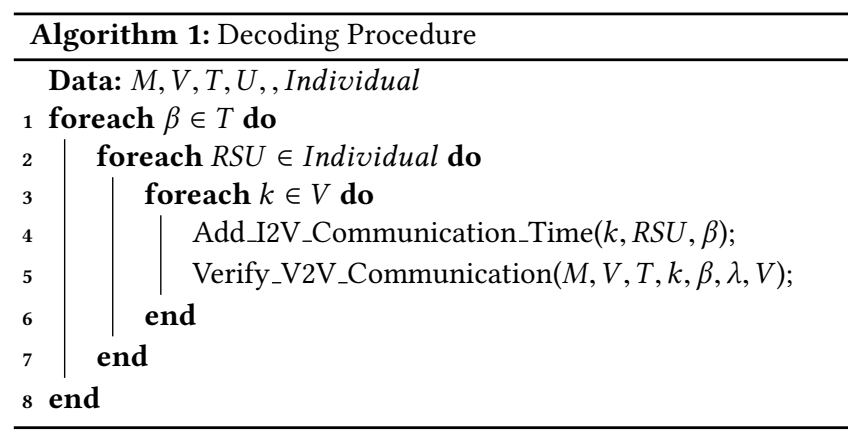

\section{EXPERIMENTS}

In this Section, we present experiments comparing our algorithm for several different $\lambda$ values (V2V communication range). Experiments are based on the realistic mobility trace (http://kolntrace.project. citi-lab.fr/) of Cologne, Germany. The Partition Program reads the original mobility trace and partition it into a grid of $100 \times 100$ urban cells.

In this work, we present the results for two different pairs of $\rho_{1}$ and $\rho_{2}$ : (i) $\Delta_{0.9}^{0.1}$; and, (ii) $\Delta_{0.1}^{0.9}$. For all instances we run the algorithm 11 times with the following parameters: $\varrho=200$, $t$ Mut $=0.4$, $t$ Cros $=0.8$ and $\tau=500$. We run our algorithm for six different $\lambda$ values $(0 \mathrm{~m}, 50 \mathrm{~m}, 100 \mathrm{~m}, 150 \mathrm{~m}, 200 \mathrm{~m}$ and, $250 \mathrm{~m})$. When $\lambda=0$, the Delta-GA2 algorithm do not use the V2V communication.

We present and compare the number of RSUs found in each tested algorithm for different $\lambda$ values. Figures $2 \mathrm{a}$ and $2 \mathrm{~b}$ present the number of RSUs for each tested scenario: In all the Figures we present the medium instance solution for 1000 and 10000 vehicles instance. For each figure, the $\mathrm{x}$-axis indicates the number of RSUs and, the $y$-axis indicates the different $\lambda$ values.

Figure 2 shows that, for the $1000 \mathrm{~V}$ trace, the number of vehicles does not decreases significantly when the $\lambda$ value increases. It happens probably because of the vehicles sparsity. As the number of vehicles is small, the number of contact opportunities seems to be rare since the communication via $\mathrm{V} 2 \mathrm{~V}$ two vehicles must be close enough from each other at the same instant of time. It is also intersting to note that for $\Delta_{0.9}^{0.1}$ when $\lambda=250$ and $|V|=1000$, the number of RSUs is smaller than when $|V|=10000$. On the other side, for 10000 vehicles instance, we can note a more significant gain. For instance, when $\rho_{1}$ and $\rho_{2}$ are equal to 0.5 , the number

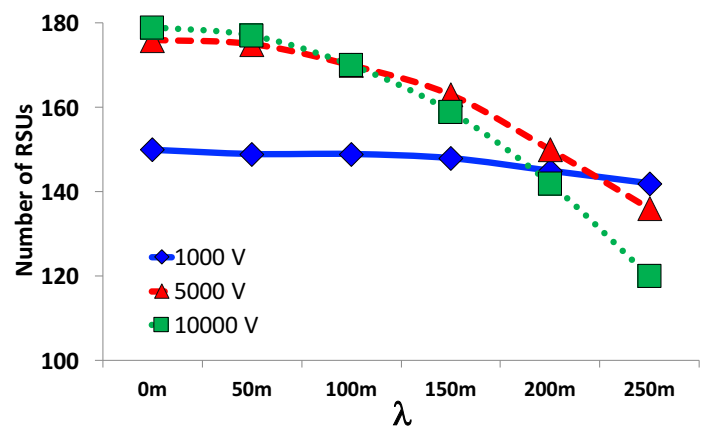

(a) Number of the RSUs $\Delta_{0.9}^{0.1}$

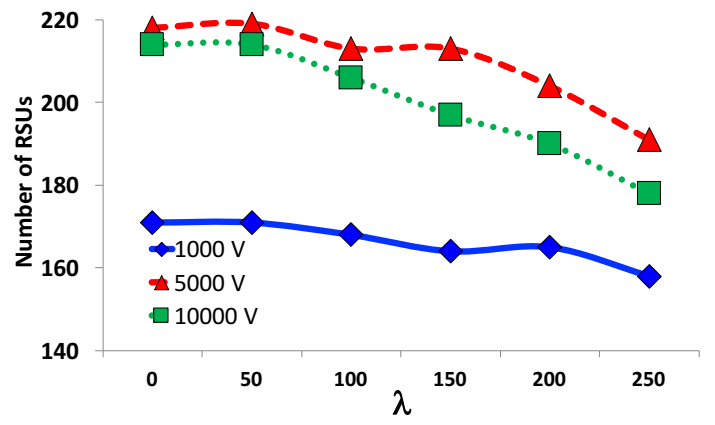

(b) Number of the RSUs $\Delta_{0.1}^{0.9}$

Figure 2: This graphic represents the number of RSUs computed by our algorithm for different $\lambda$ values. The blue solid curves, the red dashed curves and the green dotted curves represent, respectively, the number of RSUs for 2 different vehicles traces.

of RSUs decreases in $32 \%$ when we compare the number of RSUs achieved by our algorithm when $\lambda=0$ and when $\lambda=250$.

\section{FINAL REMARKS}

We noted that as bigger the $\mathrm{V} 2 \mathrm{~V}$ communication range $(\lambda)$, fewer the number of RSUs required to achieve the specific Deployment $\Delta_{\rho_{2}}^{\rho_{1}}$. Furthermore, we observed that the number of vehicles traveling the road network impacts the algorithm final results. When we have few vehicles, the contact opportunities are rarer, and it is necessary more RSUs to achieve the QoS specified by Deployment $\Delta_{\rho_{2}}^{\rho_{1}}$.

\section{REFERENCES}

[1] J.J. Blum, A. Eskandarian, and L. Hoffman. 2004. Challenges of intervehicle ad hoc networks. Intelligent Transportation Systems, IEEE Transactions on 5, 4 (Dec 2004), 347-351. DOI : http://dx.doi.org/10.1109/TITS.2004.838218

[2] H. Hartenstein and K.P. Laberteaux. 2008. A tutorial survey on vehicular ad hoc networks. Communications Magazine, IEEE 46, 6 (June 2008), 164-171. DOI : http://dx.doi.org/10.1109/MCOM.2008.4539481

[3] Melanie Mitchell. 1998. An Introduction to Genetic Algorithms. MIT Press, Cambridge, MA, USA.

[4] João F. M. Sarubbi, Flavio V. C. Martins, and Cristiano M. Silva. 2016. A Genetic Algorithm for Deploying Roadside Units in VANETs. In IEEE Congress on Evolutionary Computation (CEC), 2016 IEEE.

[5] Cristiano M. Silva and Wagner Meira. 2015. Evaluating the Performance of Heterogeneous Vehicular Networks. In Vehicular Technology Conference (VTC), 2015 IEEE. 